\title{
HR-EBSD based Characterization of Dislocations in Additive Manufactured 316L Stainless Steel
}

Tim Ruggles ${ }^{1}$, Josh Kacher $^{2}$, Matthew Nowell ${ }^{3}$ and Stuart Wright ${ }^{4}$

${ }^{1}$ Sandia National Laboratories, Albuquerque, New Mexico, United States, ${ }^{2}$ Georgia Tech School of Materials Science and Engineering, Atlanta, Georgia, United States, ${ }^{3}$ EDAX, Riverton, Utah, United States, ${ }^{4}$ EDAX, St Geprge, Utah, United States

Additive manufactured (AM) metals form complex, hierarchical dislocation structures due to the rapid cooling rates and cyclic heating/cooling cycles associated with the manufacturing process. The characteristics of these dislocation structures heavily influence the mechanical properties of the printed parts and their formation has been shown to be dependent on the processing conditions used and component geometry [1]. However, directly relating these dislocation structures to mechanical properties and print parameters has proven difficult due to the cost and time needed to accurately characterize the dislocation structures. Electron Backscatter Diffraction (EBSD) in the scanning electron microscope (SEM) is a recognized tool for analyzing both the elastic and plastic strain effects in deformed materials [2]. However, to optimize the results that obtained by EBSD for strain analysis requires higher angular resolution than can be obtained by conventional EBSD, i.e. standard automated indexing of the EBSD patterns to obtain crystallographic orientation. High (angular) Resolution Electron Backscatter Diffraction (HR-EBSD) employs cross-correlation of EBSD patterns to achieve the angular resolution needed to obtain the components of the elastic strain tensor as well as to characterize dislocation densities [3].

We have applied the HR-EBSD technique to an AM 316L austenitic stainless steel sample. EBSD patterns were obtained using an EDAX Clarity ${ }^{\mathrm{TM}}$ direct electron detector at $512 \times 512$ pixel resolution. HR-EBSD calculations were performed using OpenXY [5]. Figure 1 shows one of the shear components of the elastic strain gradient tensor for the sample investigated. The variations in elastic strain arise both from dislocations structures and from deformed regions near grain boundaries.

A detailed investigation of the dislocations associated with the three most prominent nearly parallel features in Figure 1 was undertaken using HR-EBSD. The cross-correlations used in HR-EBSD allows relative lattice distortions to be measured to approximately 200 microstrain when the relative distortions are small. These relative distortions may be related to the Nye's tensor which provides a continuum description of the geometrically necessary dislocation tensor. Nye's tensor can then be used to determine the Burgers vector and dislocation line vector at each point in the scan area [6]. Such an analysis has been applied to the AM stainless steel sample as shown in Figure 2 where the color at each point correspond to the polar coordinates of Burgers and line vectors. From these measurements, the Burgers vector for the each of three notable structures was found to vary from [110] at the left, [1-10] for the center and a mixed at right. These results demonstrate the ability to rapidly characterize dislocation structures in terms of defect character, distribution, and associated strain fields, opening up the possibility of high throughput defect structure characterization in AM parts.

This paper describes objective technical results and analysis. Any subjective views or opinions that might be expressed in the paper do not necessarily represent the views of the U.S. Department of Energy or the United States Government. Sandia National Laboratories is a multimission laboratory managed and operated by National Technology and Engineering Solutions of Sandia, LLC., a wholly owned subsidiary 
of Honeywell International, Inc., for the U.S. Department of Energy's National Nuclear Security Administration under contract DE-NA-0003525.

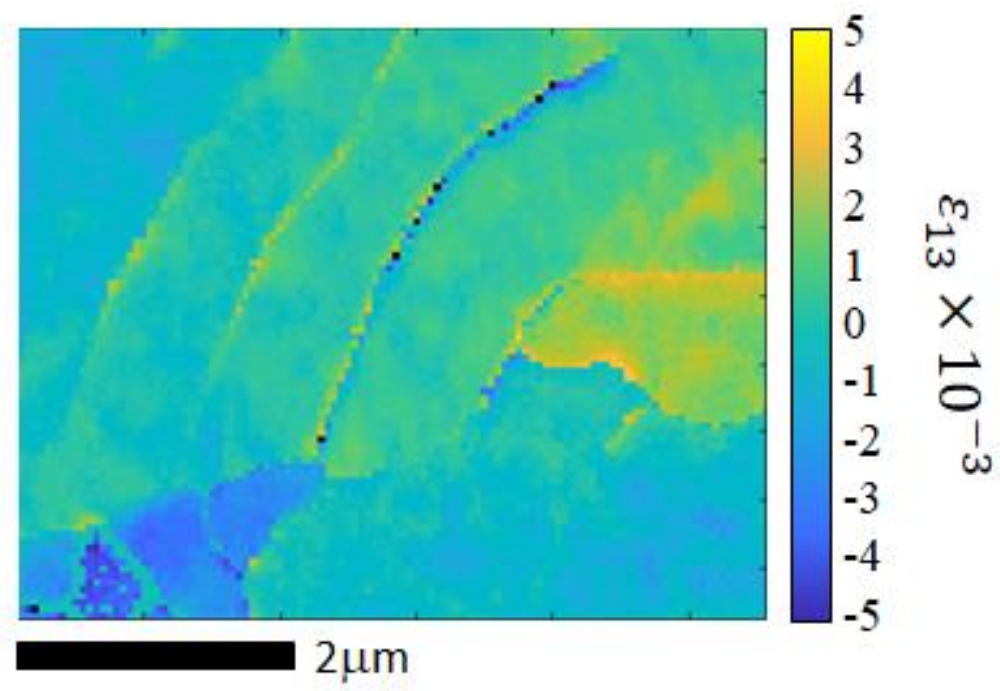

Figure 1. Elastic strain map from an AM austenitic stainless steel sample 

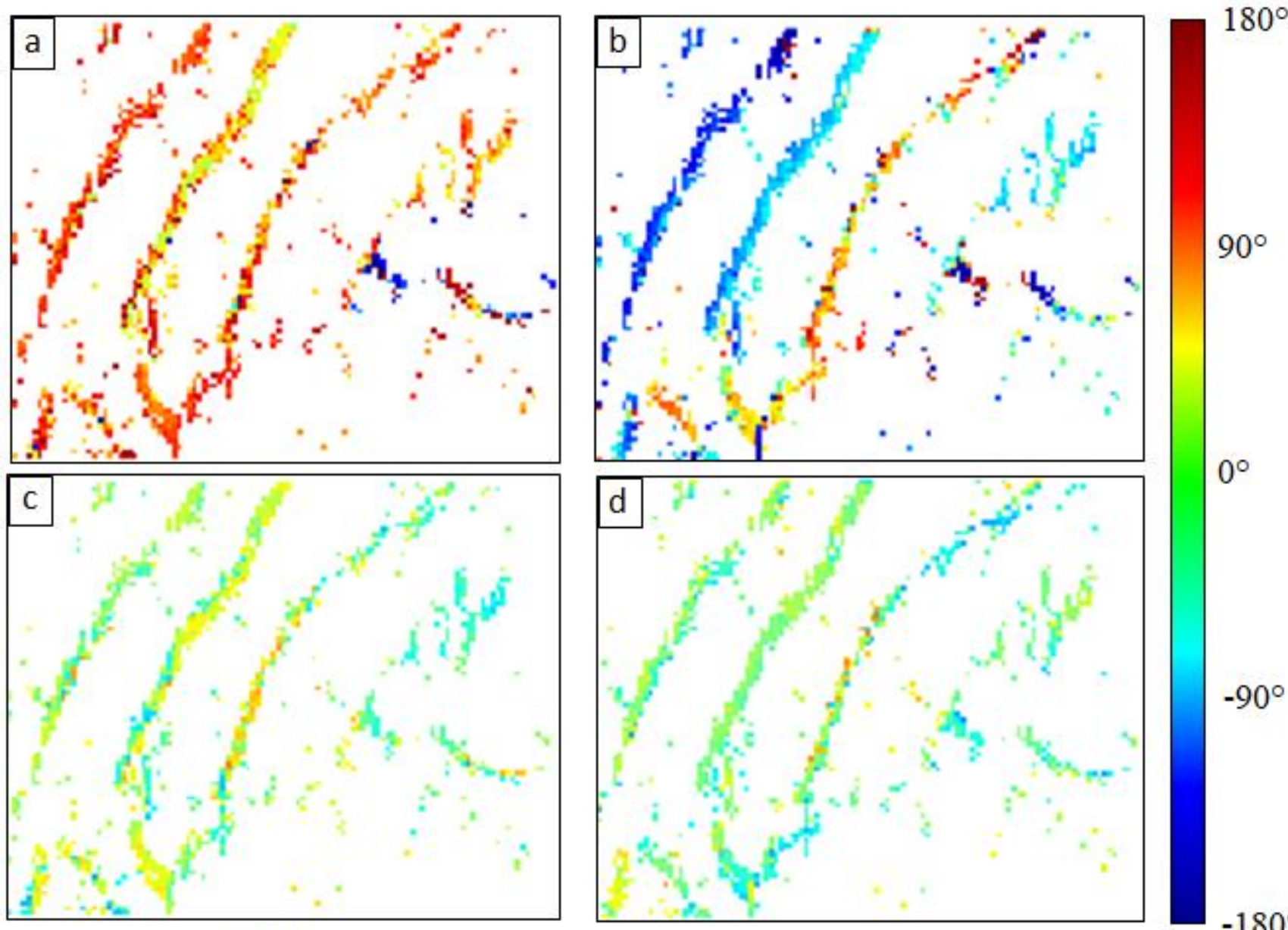

$0^{\circ}$

$2 \mu \mathrm{m}$

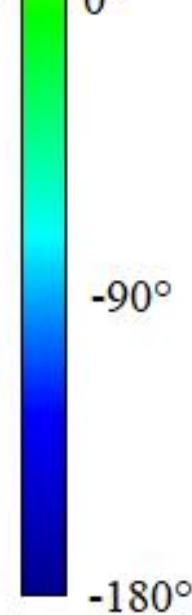

Figure 2. Polar angle plots of dislocations in an AM austenitic stainless steel sample. (a) Line vector - azimuthal, (b) Burgers vector - azimuthal, (c) Line vector - elevation and (d) Burger vector - azimuthal.

\section{References}

[1] KM Bertsch, GM de Bellefon, B Kuehl, DJ Thoma, Acta Mater. 199 (2020), p.19-33.

[2] AJ Schwartz, M Kumar, BL Adams, DP Field, Electron backscatter diffraction in materials science (Vol. 2). New York: Springer.

[3] AJ Wilkinson, G. Meaden, DJ Dingley, Mater. Sci. Tech. 22 (2006), p. 1271-1278.

[4] github.com/BYU-MicrostructureOfMaterials/OpenXY.

[5] TJ Ruggles, JI Deitz, AA Allerman, CB Carter, JR Michael, submitted (2021). 